\title{
Practical Experience in Home Care of Nursing Interns: A Qualitative Research
}

\author{
Lin Wang1, Meijuan Luo', Ruiying Huang1, Yanying Zhu' ${ }^{1}$, Zhili Chen1, Weiju Chen ${ }^{2 *}$ \\ ${ }^{1}$ Transitional Care Department, The First Affiliated Hospital of Jinan University, Guangzhou, China \\ ${ }^{2}$ School of Nursing, Jinan University, Guangzhou, China \\ Email: wanglin18903078509@126.com, *chenweiju@126.com
}

How to cite this paper: Wang, L., Luo, M.J., Huang, R.Y., Zhu, Y.Y., Chen, Z.L. and Chen, W.J. (2020) Practical Experience in Home Care of Nursing Interns: A Qualitative Research. Open Journal of Nursing, 10, 526-533.

https://doi.org/10.4236/ojn.2020.105036

Received: April 26, 2020

Accepted: May 22, 2020

Published: May 25, 2020

Copyright $\odot 2020$ by author(s) and Scientific Research Publishing Inc. This work is licensed under the Creative Commons Attribution International License (CC BY 4.0).

http://creativecommons.org/licenses/by/4.0/

(c) (i) Open Access

\begin{abstract}
Purpose: To explore practical experience in home care of nursing interns. Methods: The phenomenological method for qualitative research was to interview 15 nursing interns in a semi-structured way, and then the collected data were analyzed and summarized by using the Colaizzi analysis method. Results: Interviewing results showed that there were five recurring themes, including: 1) personalized care; 2) health education; 3) humanistic care; 4) solid professional foundation and sense of responsibility; 5) expanded vision. Conclusion: Through home care practice, nursing interns deepened their cognitions of nursing connotation, stimulated their love for nursing and motivation of continuous learning, and improved their sense of identity to nursing career. In general, the practice of home care had a positive impact on nursing interns.
\end{abstract}

\section{Keywords}

Nursing Interns, Home Care, Practical Experience, Qualitative Research

\section{Introduction}

Clinical practice stage is not only an important part of nursing career, but also the clinical starting point of nursing professional education. It affects nursing students' professional attitude, behavior skills, professional role formation and work responsibility [1]. Moreover, it is also the key period of transforming nursing knowledge into practical ability for nursing students [2]. Practice in different clinical placement departments may give different impression and motivation to nursing interns.

Home care provides medical care, disease care and life assistance to patients with impaired function, and the disabled patients or patients suffering from various diseases in their residences [3]. Home care development has been ma- 
ture in developed countries such as America and Canada [4] [5]. However, it is still in the exploring stage in China. In recent years, Transitional Care Department of the hospital where the author works has extended its services toward home care service, providing patients with high-quality nursing service. The characteristics of the service are quite different from those of the clinical departments, which is worth exploring and may bring more challenges to nursing interns. This study used phenomenological research method to understand the practical experience in home care of nursing interns, in order to provide reference for the development of educational plan of the nursing educators and the career planning of nursing students.

\section{Methods}

\subsection{Participants}

Convenience sampling method was used to interview nursing interns who practiced in Transitional Care Department of the lead author's hospital from June to October, 2019. The sample size was determined according to the data saturation principle. All interns had practical experience in other clinical departments in the hospital for more than 3 months.

\subsection{Procedures}

All participants had a face-to-face semi-structured interview, which was conducted when their internship was done in Transitional Care Department. All interviews were performed face to face by the lead author in a quiet place. The time average of the interviews was about 30 minutes, ranging from 28 minutes to 46 minutes. The sample of information saturation was appropriate. Before the interview, the lead author would introduce the purpose and significance of the research to the interviewees, and obtain interviewees' consent on the recordings. In order to protect the privacy of interns, all research contents were anonymous and interns' names were numbered instead.

\subsection{Data Collection}

Data were collected based on the phenomenological research method; and then a semi-structured interview outline was drawn up according to the purpose of the study, mainly including interns' practical experiences, their feelings and other aspects. The interview outline was as follows: 1) What do you think the difference between home care and clinical nursing in the hospital? 2) What requirements do you think nurses who provide home care should have? 3) What is the influence of home care on your professional attitude? 4) What do you learn from home care? Each question is followed by probing question such as "Please say more", "Can you take an example?". Interviewing skills were required, such as active listening and asking open-ended questions without hidden presumption. During the interview, the author tried not to interrupt the interviewees and meanwhile confirmed their opinions in time. 


\subsection{Data Analysis}

The data collection and analysis were carried out simultaneously. After each interview, two researchers transformed the interview recordings into texts within 24 hours, and then analyzed the data by using the 7-step phenomenological analysis method of Colaizzi [6], summarizing and refining the common parts of the data. Then two researchers analyzed, reviewed and corrected the research data to ensure the authenticity and objectivity of results.

\subsection{Ethical Issues}

The Ethical approval was obtained from Institutional Review Board of the author's hospital. Personal privacy may be involved in the study, so researcher adhered to the following rules when dealing with ethical issues: the purpose of the project was introduced by the researchers; recording could not be performed until the consent of the interviewees was obtained; the interviewees had the right to quit without prejudice consequences; and gratitude was showed to interviewees.

\section{Results}

\subsection{Participants}

15 interviewees were included in this study. Interviewees were between 19 and 24 years of age, with an average of $21.80 \pm 1.32$ years old. There were 2 males and 13 females, with 9 junior college students $(60 \%)$ and 6 undergraduate students (40\%). The characteristics of interviewees were presented in Table 1.

Table 1. Demographic characteristics of interviewees $(n=15)$.

\begin{tabular}{ccccc}
\hline Number & Gender (M/F) & Age (years) & Education Background & Practice Duration \\
\hline P1 & F & 21 & junior college & 5 months \\
P2 & F & 22 & undergraduate & 3 months \\
P3 & F & 23 & undergraduate & 4 months \\
P4 & M & 23 & undergraduate & 6 months \\
P5 & M & 24 & undergraduate & 5 months \\
P6 & F & 21 & junior college & 8 months \\
P7 & F & 19 & junior college & 3 months \\
P8 & F & 23 & undergraduate & 3 months \\
P9 & F & 23 & undergraduate & 4 months \\
P10 & F & 21 & junior college & 5 months \\
P11 & F & 22 & junior college & 3 months \\
P12 & F & 20 & junior college & 6 months \\
P13 & F & 22 & junior college & 4 months \\
P14 & F & 21 & junior college & 3 months \\
P15 & F & 22 & junior college & 8 months \\
\hline
\end{tabular}




\subsection{Themes}

\subsubsection{Personalized Care}

Most of the interviewees thought that home care service has a distinct feature of personalized care. P1 "My instructor paid close attention to patients, and guided them how to exercise according to their personal situation"; P5 "My instructor often make corresponding measures with different patients, which are very practical, and patients can do them well"; P8 "My instructor knew about the patient's diet in detail, so as to give them some suggestions, and teach them and their families how to do it carefully"; P13 "Compared with nursing of wards in the hospital, individualized guidance of home care is more prominent"; P15 "Home care means that nurses have more time to take care of patients and can do things better in detail".

\subsubsection{Health Education}

Health education is crucial for home-based patients, which helps patients improve their quality of life and health level [7]. Nursing interns thought that home care practice requires paying more attention to health education. P3 "Health education and guidance given by teachers are very detailed, which effectively solves patients' problems"; P4 "During delivering home care, we should be patient and careful to patients and their families, and seriously carry out health education for patients and their families"; P6 "Through the practice of home care, I realize the importance of health education for patients, and also understand that we should learn more knowledge to help patients"; P7 "Nurses in the wards focus more on specific nursing skills, while home visiting nurses pay more attention to health education"; P9 "Home care practice enables me to learn about how to carry out health education for patients".

\subsubsection{Humanistic Care}

Humanistic care ability is the core of nursing [8]. Effective humanistic care can promote patients' recovery, obtain patients' satisfaction and improve the relationship between medical staff and patients [9]. However, some nurses often neglect patients' psychology and social concerns in clinical nursing [10]. Nursing interns stated that they barely felt the humanistic care given to the patients during the clinical practice in the hospital, but they deeply felt it during home care service. P2 "What depressed me during home care practice is humanistic care, and my preceptor always warms patient's heart with words and actions"; P3 "During home care service, communication between my instructor and the patients ran through the whole process, and my instructor was very gentle and paid attention to many details"; P10 "My instructor treated patients and their families as friends, using vivid words to communicate with patients; I think it is worth to explore and learn"; P14 "My instructor always provide excellent nursing service for patients with her patience and love during home care service. In my future nursing career, I will adhere to this spirit and provide more humanistic care for patients". 


\subsubsection{Solid Professional Foundation and Sense of Responsibility}

Most of the interns thought that home visiting nurses need to be equipped with solid professional foundation, rich clinical practical experience and a high sense of responsibility. They thought that they should study hard and improve their competency to serve patients in the future. P4 "To provide home care, we need to be self-skilled and have solid professional knowledge"; P8 "Nobody can be competent for delivering home care without several years of working experience"; P11 "Home visiting nurses are required to have solid medical and nursing knowledge and rich clinical experience, and they should constantly improve their professional skills"; P12 "I will treat nursing more serious in the future by improving my professional skills and enhancing my competency"; P15 "Home care is not as simple as you thought, and it is not as typical as it was written in textbooks. There are sometimes many unexpecting conditions that you need to handle independently during home care service period. You cannot provide excellent home care service for patients if you have no sense of responsibility".

\subsubsection{Expanded Vision}

According to the internship plan of nursing students, most of the time, nursing interns practice in the hospital and seldom visit communities or patients at home. Interviewees generally thought that through home care practice, they had learned about different nursing services and different experiences. P5 "Home care practice has given me different feelings, and I like it very much"; P8 "Home care practice has brought me into contact with different nurse roles, and allowed me to learn about the extent and depth of nursing"; P11 "In the process of home care practice, I have learnt and seen some new equipment, which could not be seen in the hospital, such as home oxygenator, etc."; P12 "I have gained different nursing service modes and different patients from home care practice, which broadened my vision".

\section{Discussion}

In China, each nurse in the hospital is responsible for 8 - 10 patients [11], which is a heavy workload for nurses. All the interviewees who have practiced in clinical nursing units considered that clinical nursing service in hospital is trivial because nurses spend a lot of time on injection, drug delivery for patients and execution of doctor's orders. They do not have time to provide personalized nursing service for each patient such as health education and humanistic care which is consistent with Zheng et al. findings [12]. Based on the above situation of nursing, the connotation of nursing and the value of nurses could not be presented well. Furthermore, nurses do same things every day, making them lack of enthusiasm. As an extension of inpatient service mode, the characteristics of home care service are totally different from clinical nursing, which gives nursing students a sense of novelty, therefore it is welcomed by all nursing interns. Home care service is a one-to-one nursing service at patients' home with relatively sufficient time, which is conducive to making and implementing persona- 
lized services based on condition for each patient. In addition to providing professional nursing skills operation, home visiting nurses focus more on health education for patients and their families, the training and guidance of caregivers, and the humanistic care for patients and their families. As a result, home care can present the value and connotation of nursing in a more excellent manner. Through the practice of home care, nursing interns can fully master about the whole process of nursing services from discharge to home, and the extents and depths of nursing, so as to deepen the cognition and understanding of nursing connotation.

The professional values formed during the internship of nursing students have a crucial impact on their employment. It is an effective way to strengthen the positive guidance of nursing students' professional values for stabilizing the nursing team and reducing the loss of nursing staff [13]. During the internship of nursing students, the professional attitudes and enthusiasm of interns are affected by the clinical instructors' words and deeds [14]. Home care services are generally undertaken by advanced and capable practice nurses such as possessing solid professional foundation, comprehensive nursing skills, serious working attitudes and outstanding communication ability [15]. As an important source of knowledge and experience, instructors would set a role model for nursing interns. Influenced by these instructors, nursing interns will also have higher requirements for themselves [16]. Learning from the words and deeds from instructors, nursing interns will have a correct perspective on life and values, which will stimulate their enthusiasm, make them have a clearer understanding of nursing profession, and virtually improve their understanding of the connotation of nursing profession and their professional identification [17]. Therefore, nursing administrators of medical institutions should monitor the psychological condition of students in the course of clinical practice and take appropriate measures to regulate the negative emotions expressed by nursing students, and actively encourage them to enhance nursing students' sense of professional identity and professional self-confidence.

\section{Limitation and Future Research}

There are limitations in this study. The interview outline was limited in items and could not fully reflect the nursing interns' experience of home care practice. In the future, we will develop a questionnaire using the Delphi method based on literatures to investigate the practical experience of nursing interns more comprehensively so as to provide theoretical basis for the education and training of nursing students.

\section{Conclusion}

The practice of home care allows nursing interns to learn about the modes and service contents of nursing from other perspectives, and thus deepen their understanding of nursing connotation. This is conducive for interns to forming ho- 
listic nursing thinking, so that they can provide better services for patients in the future. Home care also supplies nursing interns for different experiences and broadly professional vision. Furthermore, having contact with excellent instructors in different positions and roles can stimulate their love for nursing profession as well as motivation for continuous learning, and improve their professional identity. The practice of home care has a positive impact on nursing interns, which is beneficial to the training of nursing professionals. It is suggested that more medical institutions create conditions and provide opportunities for nursing interns to practice home care. Meanwhile, it is recommended that the nursing administrators of medical institutions strengthen the training of clinical nurses in humanistic care literacy, personalized nursing skills, health education methods and communication skills, so as to improve the comprehensive ability of clinical nurses and the level of clinical teaching.

\section{Acknowledgements}

This study was supported by grants from Research Foundation of Department of Education of Guangdong Province (Grant NO. 2016QTLXXM_37).

\section{Conflicts of Interest}

The authors declare no conflicts of interest regarding the publication of this paper.

\section{References}

[1] McCabe, B.W. (1985) The Improvement of Instruction in the Clinical Area: A Challenge Waiting to Be Met. Journal of Nursing Education, 24, 255-257.

[2] Wang, W.T., Liu, S.X. and Bai, X. (2018) Qualitative Research on the Professional Values of Nursing Interns in Infectious Diseases Specialist Hospital. Electronic Journal of Practical Clinical Nursing, 3, 188-189.

[3] Hyde, E. and Murphy, B. (2012) Computerized Clinical Pathways (Care Plans): Piloting a Strategy to Enhance Quality Patient Care. Clinical Nurse Specialist, 26, 277-282. https://doi.org/10.1097/NUR.0b013e31825aebc1

[4] Bos, J.T., Frijters, D.H.M. and Wagner, C. (2007) Variations in Quality of Home Care between Sites across Europe, as Measured by Home Care Quality Indicators. Aging Clinical and Experimental Research, 19, 323-329. https://doi.org/10.1007/BF03324709

[5] Ogunneye, O., Rothberg, M. and Friderici, J. (2012) Nursing Home Care Quality and 30-Day Readmission Rates for Patients with Heart Failure. Circulation: Cardiovascular Quality and Outcomes, 5, A225B. https://doi.org/10.1016/j.cardfail.2012.06.012

[6] Liu, M. (2019) Application of 7 Steps of Colaizzi in Phenomenological Research Data Analysis. Journal of Nursing, 34, 90-92.

[7] Peng, L.P. (2015) Home Care for Alzheimer's Patients and Health Education for Their Caregivers. Basic Medical Forum, 19, 4104-4106.

[8] Woods, M. (2011) An Ethic of Care in Nursing: Past, Present and Future Considerations. Ethics Social Welfare, 5, 266-276. 
https://doi.org/10.1080/17496535.2011.563427

[9] Raja, S., Hasnain, M., Vadakumchery, T., Hamad, J., Shah, R. and Hoersch, M. (2015) Identifying Elements of Patient-Centered Care in Underserved Populations: A Qualitative Study of Patient Perspectives. PLoS ONE, 10, Article ID: 0126708. https://doi.org/10.1371/journal.pone.0126708

[10] Chen, H., Zheng, M.A. and Lei, M.N. (2012) Research on Caring Ability of Surgical Nurses in a 2A-Level Hospital. Journal of Nursing Administration, 12, 255-256.

[11] Fan, Y.Z, Pan, S.F. and Huang, J. (2012) Discussion and Effect on the Implementation of Tube Bed Management Responsibility System. Contemporary Medicine, 18, 125-126.

[12] Zheng, X.M., Zheng, S.L. and Che, W.F. (2004) Investigation and Analysis of Time Allocation of Hospital Nursing Activities. Chinese Journal of Nursing, 39, 915-916.

[13] Xu, J.Y., Miao, Y.X. and Yang, X.J. (2018) A study on the Influence of Reflective Practice Diary Teaching Method on the Professional Values of Nursing Students. World s Latest Medical Information Abstract, 18, 292-293.

[14] Huang, W. (2015) A Qualitative Study on the Real Experience of Three-Year Nursing Students in Different Internship Stages. Modern Medicine and Health, 31, 81-82.

[15] Chen, L. and Chen, X.Y. (2012) General Situation of Home Care at Home \& Abroad and Thoughts on the Development of Home Care in China. China Nursing Management, 12, 94-96.

[16] Li, Y.F., Zheng, J.Y., Liu, C.Q. and Chen, W.J. (2020) The Psychological Feelings of Newly Recruited Nurses on the Implementation of One-to-One Specialized Tutorial System Training: A Qualitative Research. American Journal of Nursing Science, 3, 102-106.

[17] Li, J.Q., Wen, X.L. and Zhang, Y.P. (2018) To Explore the Application of the "Growth Mentor Companion System" in the Standardized Training of New Nurses. Chinese Medical Ethics, 31, 1422-1426. 